\title{
La relación sociedad-universidad en los marcos de la globalización
}

\section{The society-university relationship in the framework of globalization}

\author{
Juan José Vizcaíno Figueroa ${ }^{1}$ \\ juanjose_vizcaino@hotmail.com
}

Recibido: 1/12/ 2016, Aceptado: 1/02/ 2017

\begin{abstract}
RESUMEN
La globalización es un fenómeno que está presente en todas las actividades sociales y que de una u otra forma influencia el desarrollo de los países y las organizaciones. La necesidad de mantener relaciones dinámicas entre los pueblos, tanto en aspectos económicos, sociales y culturales, ha contribuido a que las tecnologías de la información y la comunicación faciliten las herramientas que permitan una interacción e integración entre las personas, las instituciones y los gobiernos. En este contexto, las instituciones de educación superior, no están ajenas a las tendencias que a nivel global se manifiestan, principalmente en su rol impulsor de una sociedad del conocimiento, que promueve la innovación, el emprendimiento y el desarrollo económico. Para tal efecto, es imperioso entender la dinámica en la cual se debe llevar a cabo la relación sociedad-universidad, comprendiendo que es una relación de doble vía, que coadyuva al desarrollo de un país, principalmente por la pertinencia de su oferta académica y la responsabilidad social que conlleva su accionar. Es por ello que el objetivo de este trabajo, es aportar con elementos de análisis y reflexión, sobre cómo la globalización y la sociedad del conocimiento permiten reorientar la forma de relacionar la sociedad y la universidad.
\end{abstract}

Palabras clave: Globalización, Vinculación con la sociedad, Gestión universitaria

\begin{abstract}
Globalization is a phenomenon that is present in all social activities and that in one way or another influences the development of countries and organizations. The need to maintain dynamic relations between peoples, in economic, social and cultural aspects, has contributed to the information and communication technologies providing the tools that allow interaction and integration among individuals, institutions and governments. In this context, institutions of higher education are not alien to the trends that are manifested globally, mainly in its role as a driver of a knowledge society, which promotes innovation, entrepreneurship and economic development.
\end{abstract}

\footnotetext{
1 Magíster en Diseño y Evaluación de Proyectos de la Universidad Central del Ecuador, Candidato a Doctor en Ciencias Económicas de la Universidad de La Habana. Desde el 2012, vinculado al proceso de suspensión y liquidación de Instituciones de Educación Superior (IES). En el 2017, Especialista en Temas de Postgrado en el Consejo de Educación Superior. Ecuador.
} 


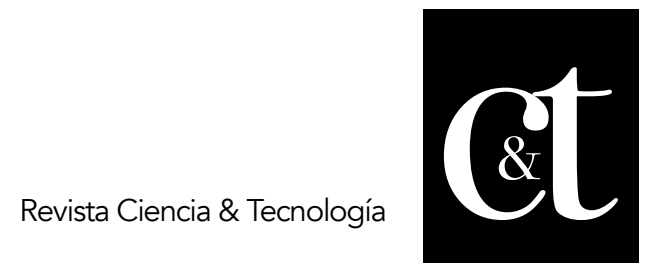

No. 14, 30 de abril de 2017

ISSN impreso: 1390 - 6321

For this purpose, it is imperative to understand the dynamics in which the societyuniversity relationship must be carried out, understanding that it is a two-way relationship that contributes to the development of a country, mainly due to the pertinence of its academic offer and the Social responsibility that entails their actions. This is why the objective of this work is to contribute with elements of analysis and reflection, on how globalization and the knowledge society allow to reorient the way of relating society and the university.

Keywords: Globalization, Relationship with society, University management

\section{Introducción}

La globalización es un fenómeno que está presente en todas las actividades sociales y que de una u otra forma influencia el desarrollo de los países. La necesidad de mantener relaciones dinámicas entre los pueblos, tanto en aspectos económicos, sociales y culturales, ha contribuido a que las tecnologías de la información y la comunicación faciliten las herramientas que permitan una interacción e integración entre las personas, las empresas y los gobiernos.

En este contexto, se incluyen la generación de conocimientos y la ciencia en sí misma, que se generan en la actividad universitaria, la misma que en el Ecuador, constituye un eje estratégico que el Estado está impulsando con fuerza, mediante una reestructuración total de los organismos que la rigen, pero más importante aún, desde un enfoque amplio que se maneja a partir de la Constitución, que en su artículo 350 señala que "El sistema de educación superior tiene como finalidad la formación académica y profesional con visión científica y humanista, la investigación científica y tecnológica, la innovación, promoción, soluciones para los problemas del país, en relación con los objetivos del régimen de desarrollo" (Art. 350).

Además, el Sistema de Educación Superior, debe regirse por los principios de autonomía responsable, cogobierno, igualdad de oportunidades, calidad, pertinencia, integralidad, autodeterminación para la producción del pensamiento y conocimiento, en el marco del diálogo de saberes, pensamiento universal y producción científica tecnológica global (Art. 351). El Estado, juega un rol decisivo para que este sistema funcione, debiendo impulsar el desarrollo de una sociedad del conocimiento para alcanzar los objetivos nacionales, promoviendo la generación, producción, difusión y acceso a los conocimientos científicos y tecnológicos.

En el Ecuador, ha sido imperante la necesidad de rescatar el rol del Estado en la dirección, regulación y supervisión de las Instituciones de Educación Superior (IES), que se perdió en forma creciente en las décadas de los gobiernos neoliberales, que con sus políticas sobre educación superior, relegaron la creación de universidades y escuelas politécnicas, así como su oferta académica, a las fuerzas del mercado, convirtiendo a la educación en una mercancía, al estudiante en cliente y a la universidad en empresa.

Este escenario descrito, contribuyó a una fragmentación de las instituciones de educación superior (IES) ecuatorianas, donde sus conceptos y modelos de gestión, posibilitaron la implementación indiscriminada de prácticas empresariales como la contratación de docentes por horas, el trato clientelar a estudiantes y la escasa importancia a la generación de ciencia y tecnología. Es por eso que el objetivo de este trabajo, es analizar los conceptos de globalización, sociedad del conocimiento y como éstos contribuyen a reorientar el sentido de pertinencia y de responsabilidad social de las instituciones de educación superior ecuatorianas, planteando una nueva forma de 


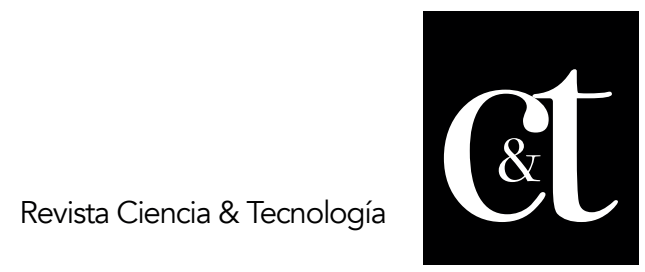

No. 14, 30 de abril de 2017

ISSN impreso: 1390 - 6321

relacionar la universidad con la sociedad.

\section{La globalización y la sociedad del conocimiento}

En la crisis actual, el capitalismo intenta volver a su utopía permanente, la de la sumisión de la vida social a la lógica exclusiva del mercado y a la globalización sin freno. Todas las regiones del planeta se encuentran ya enfrentadas a este mismo problema, pero bajo condiciones extremadamente diferentes.

El término globalización tiene una serie de definiciones, para algunos, como el Banco Mundial $o$ el Fondo Monetario Internacional, su significado se centra en la interdependencia económica que existe entre los países del mundo, resultado del aumento del volumen y la variedad de transacciones de bienes y servicios realizados fuera de sus fronteras. Para otros, como la CEPAL (2002), Santamarta (s/f) y Bonnet (2002), no es más que la desaparición de fronteras geográficas, materiales, políticas y culturales, que posibilitan la combinación de procesos comerciales y financieros.

Amin (2008:1), describe a la globalización como la lógica de expansión del capitalismo y la dimensión imperialista de su despliegue, debido a que plantea que la globalización se "impondría a todos los países de la misma forma, sea cual sea su opción de principio, capitalista o socialista, y que actúa entonces como una ley de la naturaleza producida por el estrechamiento del espacio planetario". La globalización aparece "con la onda expansiva del neoliberalismo, con su programa del Estado desertor" (Borja, 2002: 1), donde se marcó la derechización de la política y se privatizaron los servicios en forma indiscriminada.

El Banco Mundial, menciona que la globalización no es una tendencia del todo nueva, puesto que, en los tiempos remotos de la historia humana, las poblaciones se encontraban dispersas y mantenían procesos de intercambio económico y comercial, marcando una tendencia que hoy se ha consolidado en la mayoría de países. Sin embargo, Amin (2008), establece una diferencia importante entre el proceso de la antigüedad y la globalización de los tiempos modernos, al mencionar que en tiempos pasados se ofrecían oportunidades a las regiones más atrasadas para que pudieran acercarse al nivel de desarrollo de las más avanzadas, las mismas que eran tomadas o desechadas, dependiendo exclusivamente de sus propios sistemas políticos, ideológicos y culturales, de acuerdo a sus capacidades para enfrentar los desafíos que significaban las regiones más avanzadas.

La globalización de los tiempos modernos es polarizante ya que está asociada al capitalismo, donde la propia lógica de su expansión global, produce una desigualdad creciente entre quienes participan del sistema. Esto quiere decir que la globalización no ofrece oportunidades, sino que requiere la implementación de políticas voluntarias que entran en conflicto con las lógicas unilaterales de la expansión capitalista, obligando a los actores a ajustarse a las exigencias del propio desarrollo.

Borja (2002), en su conferencia dictada en ocasión de recibir el título de Doctor Honoris Causa de la Universidad Nacional de Córdoba, Argentina, describe a la globalización como un hecho económico y plantea el globalismo como la teoría que lo justifica, que defiende el fundamentalismo del mercado y el libre comercio. Es por eso que antes de abordar la relación que existe entre la globalización y la sociedad del conocimiento, es importante mantener la línea trazada por el ex presidente de la República del Ecuador, Dr. Rodrigo Borja, acerca de comprender el globalismo como la teoría que justifica y estimula la globalización. En ese sentido, Ianni (1999) menciona que con el desarrollo intensivo y extensivo del capitalismo surge una 


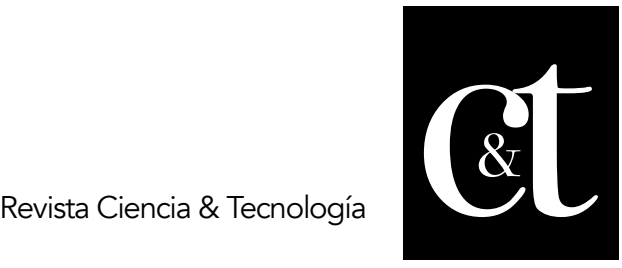

No. 14, 30 de abril de 2017

ISSN impreso: 1390 - 6321

configuración geo-histórica dotada de peculiaridades específicas y de movimientos propios, que puede denominarse globalismo.

La humanidad ha atravesado diversos procesos histórico-sociales que han marcado las relaciones económicas, sociales, políticas y culturales de diferentes épocas, tales como el feudalismo, esclavismo, mercantilismo, colonialismo, imperialismo, capitalismo y socialismo. El globalismo es un proceso histórico-social de una realidad múltiple presente en el ámbito transnacional que, como todo proceso, no anula al preexistente sino transforma el mapa del mundo, no solo en la geografía e historia sino también en las conciencias de las personas.

Smith (2001), en su artículo "Nuevo globalismo, nuevo urbanismo", plantea dos líneas de pensamiento que enmarcan los argumentos actuales del globalismo, la primera la denomina "el final de la geografía", que al igual que los otros autores que se han citado, defiende el rompimiento del espacio, lugar y límites, debido al auge de los nuevos mercados financieros y el avance de las tecnologías de la información, que coadyuvan al desarrollo económico donde la situación geográfica ya no afecta a las finanzas, convirtiendo al mundo en un espacio de flujos más que en un espacio de lugares.

La segunda corriente defiende una marcada reestructuración de los conceptos de espacio, lugar y geografía. El lugar ocupa una nueva importancia en esa visión de nuevo globalismo, ya que "resulta central para la circulación de personas y de capital que constituyen la globalización, y la centralidad de los ámbitos urbanos en un mundo globalizado trae consigo el reconocimiento de la rápida pérdida de importancia de la economía nacional" (Smith, 2001: 24).

En tal virtud, se puede comprender de mejor manera la polarización que se dio entre producción y finanzas, debido a que el lugar donde se desarrollaba cada uno, establecía brechas entre clases sociales por los puntos de concentración de sus actividades. Frente a esta realidad, surge la denominada sociedad del conocimiento, que Krüger (2006: 2) manifiesta que sus orígenes datan a los años 60, cuando Peter Drucker estableció como características de este tipo de sociedad, "una estructura económica y social en la que el conocimiento sustituye al trabajo, a las materias primas y al capital como fuente más importante de la productividad, crecimiento y desigualdades sociales".

La Organización de los Estados Americanos se refiere a la sociedad del conocimiento a aquella que está bien educada y que se basa en el conocimiento de las personas para promover la innovación, el emprendimiento y el desarrollo económico. Globalización y sociedad del conocimiento son los ejes estratégicos del siglo XXI. En los últimos años la interacción de ambos y, en su momento influyendo por su propia cuenta, han conformado un marco que explica en buena parte el crecimiento económico y el progreso en general. Constituye una tarea prioritaria para estudiosos y analistas, el comprender de mejor manera, ambos fenómenos y tratar de explotar sus ventajas para la educación superior.

\section{Pertinencia y responsabilidad social de la universidad}

De lo dicho en párrafos anteriores se desprende el hecho de reorientar y reafirmar el sentido de pertinencia que deben tener las IES para contribuir al desarrollo de una sociedad. Según Pérez (2007) El análisis de la proyección social de la educación es objeto de estudio de La Sociología de la Educación y se caracteriza por considerar: 


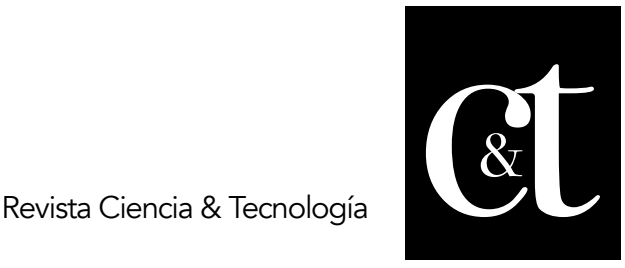

No. 14, 30 de abril de 2017

ISSN impreso: 1390 - 6321

- La incidencia de la educación en el dinamismo general de la sociedad,

- La educación como el factor principal del proceso de socialización de los individuos,

- El papel de la educación en el control social, en la reproducción social, en la movilidad social, en la selección social y adaptación social.

La misma autora señala que "La pertinencia social relaciona a la Educación Superior con la Sociedad planteando la necesidad de una evaluación externa en términos de expectativas y logros públicos" y que "El concepto de pertinencia obliga a la Universidad a responder ante la sociedad sobre lo que la institución universitaria genera como producto" (Pérez, 2007: 4).

Para García (1997) la pertinencia está vinculada a una de las principales características que tiene el nuevo contexto de producir conocimientos, esto es, el énfasis en tomar en cuenta el entorno en el cual están insertas las instituciones de investigación y, por lo tanto, la necesidad de un estrecho acercamiento entre los que producen y entre los que se apropian del conocimiento. Por un lado, los que se apropian, o sea los usuarios del conocimiento, son no solamente los estudiantes, 0 usuarios internos, sino las comunidades en la que están insertas las instituciones, y también, de manera muy importante, los otros niveles del sistema educativo.

Desde este punto de vista la pertinencia, en la medida misma en que se sitúa en el horizonte de un responder a la sociedad, se vincula con el principio de responsabilidad, y permite de este modo romper la posibilidad de un cerco de autoaislamiento universitario incompatible con la misma tradición latinoamericanista de una universidad en armonía con las necesidades de la sociedad civil y con las condiciones de su progreso (Pérez, 2007) De aquí parte la responsabilidad social universitaria, que se puede entender como el "compromiso institucional avocado hacia los miembros de una determinada comunidad, de manera personal o colectiva para trabajar en bien de los recursos comunes, que hacen de compartir la vida, una conciencia pacífica y proactiva" (Orozco y Astudillo, 2011: 91).

Para conseguir este cometido, "Al igual que la Empresa que ha debido superar el enfoque filantrópico de la inversión social (como gasto extra) para entenderse a sí misma bajo el nuevo paradigma de la Responsabilidad Social, la Universidad debe tratar de superar el enfoque de la "proyección social y extensión universitaria" como "apéndices" bien intencionados a su función central de formación estudiantil y producción de conocimientos, para poder asumir la verdadera exigencia de la Responsabilidad Social Universitaria" (Vallaeys, 2008: 2).

Su responsabilidad social, por ende, no consiste simplemente en el responder ante, sino también en el debate que la universidad es susceptible de generar sobre el marco filosófico-político que condiciona la negociación con el estado sobre la pertinencia de lo que la universidad produzca. Es por eso que la universidad debe a la vez hacerse cargo de lo que ella es como especificidad institucional y rendir cuentas a la sociedad de lo que ella hace y produce como bien público (Pérez, 2007).

En este mismo sentido, Camacho, Fernández y Miralles (citados en Orozco y Astudillo, 2011), se refieren a que la responsabilidad social es a la vez una exigencia ética y una estrategia racional de desarrollo para la inteligencia organizacional que debe considerarse que al contar con la participación de todos los actores comunitarios se estará logrando articular mecanismos que posibiliten el desarrollo autónomo de cada 


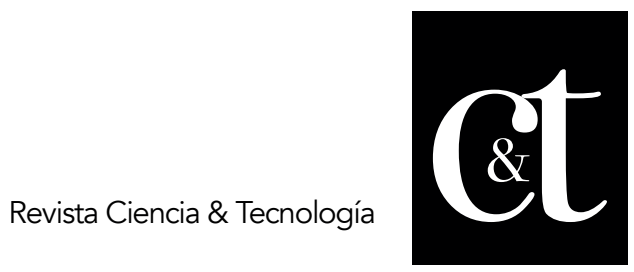

No. 14, 30 de abril de 2017

ISSN impreso: 1390 - 6321

localidad.

La UNESCO (2009), en su Declaración de la conferencia Mundial de la Educación Superior, también hace referencia a la responsabilidad social de la educación superior manifestando que las instituciones de educación superior a través de sus funciones principales que son: la investigación, la enseñanza y el servicio a la comunidad llevados adelante en el contexto de la autonomía institucional, la libertad académica debería aumentar su foco interdisciplinario y promover pensamiento crítico y activar ciudadanía que contribuya al desarrollo y al avance de la sostenibilidad del desarrollo, la paz, el bienestar, el desarrollo y la realización de los derechos humanos incluyendo la equidad y género.

Los desafíos entonces, están en aproximar la universidad a la sociedad, en hacerla pertinente para sí misma y para la sociedad, en comprobar como esta universidad se inserta en el contexto que la contiene y cómo responde a las expectativas y exigencias sociales con acciones comprometidas, relevantes y oportunas.

Una nueva visión de la interacción sociedad-universidad luego del estruendoso fracaso del neoliberalismo y más recientemente por la crisis mundial del capitalismo, se perfila una concepción con prácticas democráticas y participativas, que rescatan valores como el amor, la solidaridad y la igualdad. Son precisamente este tipo de prácticas las que impiden que el socialismo del siglo XXI, como se denomina a esta tendencia, surja por decisión de un gobierno, de una vanguardia iluminada. "No puede decretarse desde arriba. Es un proceso que se construye con la gente, en la que esta, al ir transformando las circunstancias, se transforma así misma. No es una dádiva, es una conquista" (Harnecker, 2012: 4).

Lamentablemente en la actualidad, se puede observar que en los países donde se ha implementado este socialismo del siglo XXI, han vuelto las prácticas soviéticas que tan duramente han sido criticadas, como ejemplo, el totalitarismo, la falta de democracia, la planificación central burocrática, el capitalismo del Estado, la pretensión de homogenizar sin respetar las diferencias, la producción sin tener en cuenta la necesidad de preservar la naturaleza, entre otros rasgos de aquel modelo que no tuvo éxito en varios países.

Sin embargo, de estos problemas que se empiezan a suscitar, no cabe duda que este modelo plantea cosas importantes, como poner a la persona sobre el capital, orientando los servicios hacia la satisfacción de necesidades humanas y no a la obtención de lucro. De aquí se desprende la importancia de plantear una visión de la educación superior como servicio y derecho.

Para lograr este cometido se debe establecer una relación entre la academia y la sociedad. Esta vinculación con la comunidad ha sufrido varios cambios de enfoque a lo largo de los años, que han permitido orientar de mejor manera el trabajo que las IES deben hacer como aporte al desarrollo de la sociedad.

La extensión universitaria, fue la primera forma de acercar la universidad a la sociedad. Este término "se empleó por primera vez en 1873, en la Universidad de Cambridge, Inglaterra, para designar una determinada innovación en materia de enseñanza. Se trataba de poner las ventajas de la formación universitaria al alcance de toda la gente, en los sitios donde vivía y trabajaba" (Pérez, 2005: 22).

En América Latina en cambio, González (2002) señala que este concepto nace en el año 1918 con la Reforma de Córdoba en Argentina, a raíz del debate sobre el sentido 
social de la universidad, despreocupada hasta entonces de la realidad y sus problemas. Surge entonces como una función sustantiva como la docencia o la investigación.

El movimiento de Córdoba tuvo como protagonista a la clase media emergente y en cuyo manifiesto se establece que las universidades deben hacer Extensión Universitaria, fortalecer la función social, proyectar al pueblo la cultura universitaria y preocuparse por los problemas nacionales (Armas et al., 1984).

A partir de los años 70 con la Cumbre de la Tierra, celebrada en Estocolmo en 1972 se abre un nuevo período histórico en el desarrollo de la Extensión Universitaria yen fin de todas las organizaciones, instituciones y entidades que fungen como actores sociales del desarrollo; la función de extensión se constituye como un pilar importante para el desarrollo social de los pueblos en un momento histórico en que el Estado Nación tiende al debilitamiento por diferentes vías, entre ellas el proceso de transnacionalización del capital y el flujo migratorio sur norte. De aquí se derivan los estatutos de la mayoría de la Universidades Latinoamericanas donde se plantea que la Extensión Universitaria tiene como objetivo promover la cultura nacional, incidir en la integración, coadyuvar a los planes de desarrollo regional y territoriales, sumarse a la lucha por la liberación nacional en compromiso con las clases populares (Pérez, 2005).

Sin embargo, los escasos avances en la vinculación dentro de las instituciones de educación superior latinoamericanas se deben a la carencia de un significado claro y socialmente compartido sobre lo que es esta función universitaria. Lo que se ha venido manejando en la universidad ecuatoriana y en general en América Latina en torno a la vinculación es una visión economicista que no ha permitido reconocerla como una función sustantiva adicional a las que habitualmente se reconocen en las IES.

Lo que ha prevalecido es la vinculación especialmente con el sector productivo, con el que se firman un sin número de convenios de colaboración pero que no han generado los resultados esperados.

Frente a esta situación, Campos y Sánchez Daza (2005) plantean que estos acercamientos con la planta productiva o con la sociedad se han hecho a partir de un desconocimiento, especialmente teórico, de la función de vinculación determinado por una confusión con otras actividades, básicamente las de extensión y las de prestación de servicios.

Los mismos autores sostienen que es muy reciente el reconocimiento dentro de las propias universidades, de que ésta es una función que implica profesionalización y que administrativamente, las oficinas de gestión de la vinculación están ubicadas en estructuras de tercer o cuarto nivel y, en muchas ocasiones, dependen de áreas cuya función no tiene nada qué ver con esta actividad.

Es por eso que el punto de partida debe tener un concepto claro de lo que es realmente la vinculación con la comunidad y a partir de éste, orientar los esfuerzos hacia la elaboración de un modelo de gestión eficiente.

Frente a este reto de conceptualizar la vinculación, hay que diferenciar las visiones desde tres posiciones (Campos y Sánchez Daza, 2005): 


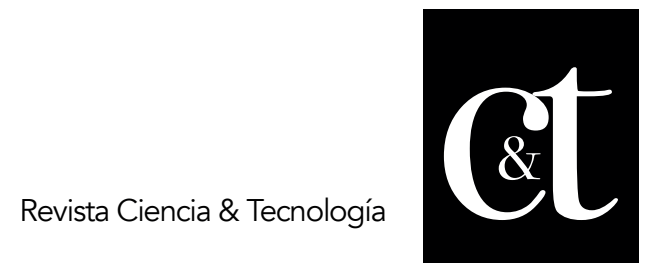

No. 14, 30 de abril de 2017

ISSN impreso: 1390 - 6321

- Quienes consideran que la vinculación tiene un contenido básicamente económico.

- Quienes consideran que la vinculación se resuelve exclusivamente mediante un acercamiento físico con la sociedad (visión fisicalista, que también está fuertemente asociada a una visión asistencial).

- Quienes consideran que la vinculación es una nueva función sustantiva de las Universidades.

Varios autores aportan a la comprensión conceptual de lo que es la vinculación, uno de esos conceptos es que la vinculación es:

El medio que permite a la universidad interactuar con su entorno, coordinando eficaz y eficientemente sus funciones de docencia, investigación y extensión de la cultura y los servicios, a la vez que favorece su capacidad de relacionarse con los sectores productivo y social en acciones de beneficio mutuo, lo que favorece su posicionamiento estratégico (Alcántar y Arcos, 2004, p. 6).

El Consejo Nacional de Evaluación y Acreditación de la Educación Superior del Ecuador (2009) estableció como la definición de la vinculación en su Modelo de Evaluación de Desempeño Institucional de las Instituciones de Educación Superior la "interacción de la institución con los demás componentes de la sociedad, para mutuo beneficio en el avance del conocimiento, la formación de recursos humanos y la solución de problemas específicos en función del desarrollo" (CONEA, 2009: 53).

Pérez (2007:6) la define como "la función que le permite a la Universidad interactuar proactivamente con su entorno social, dimensión del proceso educativo que se articula transversalmente con las restantes funciones y subsistemas de la Universidad". Todo ello para lograr una nueva cualidad en el desarrollo sociocultural de las comunidades intra y extra muros al promover cultura, ciencia, técnica y valores que comprometen al hombre con la sociedad.

Para Campos y Sánchez Daza (2005:10), la vinculación como nueva función de las universidades es "un eje estructurador de la planeación académica, esto es, que las funciones de docencia e investigación universitarias encuentran mecanismos y formas de articulación de manera más estrecha y efectiva con la sociedad y la economía, salvando el carácter asistencial que hasta antes prevalecía".

Esta nueva visión de la vinculación permite que las funciones universitarias interactúen entre sí posibilitando un trabajo armónico y efectivo con la comunidad, Segredo (2011:167) las define así:

"- La docencia es la función por la que se trasmite en el proceso académico los conocimientos, se dirige la aplicación de estos a la realidad espacio/temporal y se desarrolla la actitud heurística.

- La investigación se orienta hacia la búsqueda de conocimientos nuevos y a la comprobación de los ya existentes. Su meta es la superación del acervo existente, el entrenamiento de los estudiantes/profesionales en los métodos del conocimiento y actuar sobre los problemas sociales prioritarios.

- La extensión es la función que vincula entre sí el conocimiento y el ámbito de la realidad y del universo.

- Su misión es extender hacia la sociedad la actividad orgánica de la Universidad, integrando en aquella el modelo de práctica profesional multifacética que postula la filosofía institucional, devolviéndole parte de sus aportes y recogiendo del seno vivo 
de su universo las orientaciones del saber popular".

Adicionalmente, el mismo autor plantea que hay que entender que la gestión universitaria y los procesos administrativos deben estar presentes en cada función universitaria y que se condicionan por los siguientes criterios:

"- El crecimiento de la escala de actividad de estas instituciones, que incrementa significativamente la complejidad de su gestión.

- El vínculo cada vez más estrecho entre los productos de la educación superior y el desarrollo económico y social de cada país que evidencia la exigencia sobre su eficacia.

- El incesante aumento de los costos de la educación superior y la disminución en la afluencia de recursos a las IES, que ha provocado un interés marcado en la racionalidad de los procesos y en la utilización de los recursos.

- La necesidad de integración del trabajo docente y científico (interdisciplinariedad, transdisciplinariedad) y su proyección institucional como trabajo cooperativo" (Segredo, 2011: 165).

Con todos estos elementos queda claro que la vinculación es una función que permite a las universidades realinear sus objetivos y visiones a futuro, sin dejar de tener los pies sobre la tierra ni de reconocerse como una parte más de la sociedad. Ayuda a las universidades a identificarse como instituciones interesadas en participar en la solución de las problemáticas que enfrentan los ciudadanos de las regiones en las cuales están localizadas o de la sociedad en general.

Campos y Sánchez Daza (2005:11) manifiestan que "Al igual que el resto de funciones universitarias, ésta debe integrarse a la cotidianidad académica y ser resuelta de manera colectiva. Las oficinas de vinculación sólo asumen el papel de "facilitadoras" de esta actividad que día a día cultivan y consolidan los académicos e investigadores de cada facultad o centro de investigación".

Además, estos mismos autores sostienen que "Esta nueva propuesta incluye también aspectos fundamentales como: la inclusión de la evaluación de la propia vinculación; la vinculación no sólo al exterior sino, sobre todo, al interior de la propia Universidad" (Campos y Sánchez Daza, 2005: 11).

\section{Conclusiones}

Los modelos de gestión universitarios no están ajenos a la influencia de los fenómenos económicos y sociales que se desarrollan a nivel global. La necesidad de adaptarse a los cambios que se producen a diario debe ser una prioridad para las autoridades universitarias. No debe perderse de vista la misión principal de la educación superior, que es formar profesionales que contribuyan al desarrollo de la sociedad apoyándose en la generación de ciencia y tecnología.

El Ecuador ha dado pasos firmes en torno a conseguir este cometido, introduciendo conceptos y lineamientos generales en las Leyes que rigen al país, coadyuvando a que las instituciones de educación superior planifiquen y desarrollen sus actividades en relación con los objetivos nacionales. Se debe consolidar la interacción sociedaduniversidad, con el objetivo de promover la pertinencia de la oferta académica y de que se instauren modelos de gestión que permitan mejorar la calidad académica e investigativa de las Instituciones de Educación Superior ecuatorianas enmarcadas en 


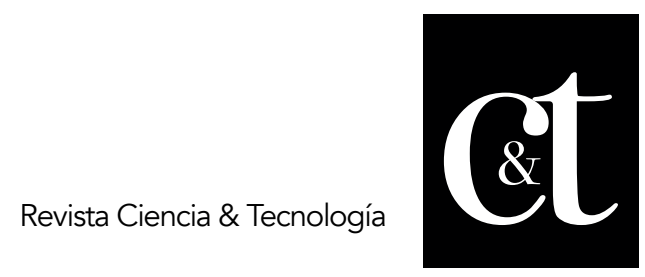

No. 14, 30 de abril de 2017

ISSN impreso: 1390 - 6321

las tendencias internacionales y necesidades locales de desarrollo.

\section{Referencias bibliográficas}

Alcántar, M. y Arcos, L. (2004). La vinculación como instrumento de imagen y posicionamiento de las instituciones de educación superior. Revista Electrónica de Investigación Educativa, 6 (1).

Amin, S. (2008). Capitalismo, imperialismo, mundialización. Instituto Argentino para el Desarrollo Económico. Consultado el 15 de enero de 2014 en http://www.iade.org.ar/modules/noticias/article.php?storyid=2217.

Armas, R., Torres, E., Cairo, A. (1984). Historia de la Universidad de la Habana. 19301978. Editorial de Ciencias Sociales, 2.

Banco Mundial, consultado el 29 de diciembre de 2013 en http://www.bancomundial.org/temas/globalizacion/cuestiones1.htm.

Bonnet, A. (2002). La globalización y las crisis latinoamericanas. Revista Mensual de Economía, Sociedad y Cultura. Consultado el 10 de enero en http://rcci.net/globalizacion/index.htm.

Borja, R. (2002). Educación, globalización y sociedad del conocimiento. Conferencia dictada en ocasión de recibir el título de Doctor Honoris Causa, Universidad Nacional de Córdoba.

Campos, G. y Sánchez Daza, G. (2005). La vinculación universitaria: Ese oscuro objeto del deseo. Revista Electrónica de Investigación Educativa, 7 (2).

Castelló. J. M., Garrigós, J. A. y Aparisi, A. M. (2007). Introducción a la organización y gestión de empresas. Valencia: UPV.

CEPAL (2012). Globalización y desarrollo (Síntesis). Vigésimo noveno Período de Sesiones. Brasil del 6 al 10 de mayo 2002.

Consejo Nacional de Evaluación y Acreditación de la Educación Superior del Ecuador. (2009). Evaluación de Desempeño Institucional de las Instituciones de Educación Superior. Mandato Constituyente No. 14.

Consejo Nacional de Evaluación y Acreditación de la Educación Superior del Ecuador. (2009). Modelo de Evaluación de Desempeño Institucional de las Instituciones de Educación Superior. Mandato Constituyente No. 14.

Constitución Política de la República del Ecuador. 2008.

Fondo Monetario Internacional, consultado el 29 de diciembre de 2013 en http://www.imf.org/ external/np/exr/ib/2000/esl/041200s.htm.

García, C. (1997). El valor de la pertinencia en las dinámicas de transformación de la educación superior en América Latina. La educación superior en el Siglo XXI. Visión de América Latina y el Caribe. Tomo I. Colección Respuestas. Ediciones CRESALC/UNESCO, Caracas.

González, M. (2002). Un Modelo de gestión de la extensión universitaria para la 


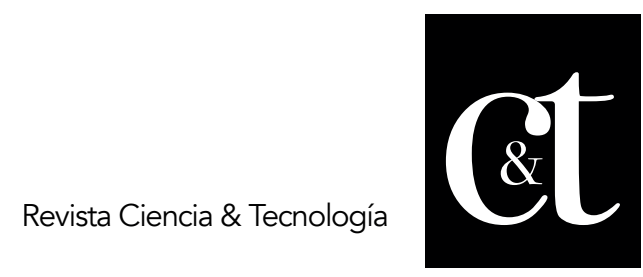

No. 14,30 de abril de 2017

ISSN impreso: 1390 - 6321

Universidad de Pinar del Río. Tesis doctoral, Universidad de Pinar del Rio.

Harneccker, M. (2012). Cinco reflexiones sobre el socialismo del siglo XXI. Science and Society, 76 (2). Traducción.

Ianni. O. (1999). La era del globalismo. Nueva sociedad. No. 163, 92-108.

Krüger, K. (2006). El concepto de sociedad del conocimiento. Revista Bibliográfica de Geografía y Ciencias Sociales. Universidad de Barcelona, Vol. XI, No.683. Consultado el 20 de febrero de 2014 en http://www.ub.es/geocrit/b3w-683.htm.

Organización de los Estados Americanos, consultado el 18 de febrero de 2014 en http://www. oas.org/es/temas/sociedad_conocimiento.asp.

Orozco, J., Astudillo, A. (2011). Enfoque de gestión universitaria. Revista Ciencia UNEMI (6), 88-96. ISSN: 1390-4272.

Pérez, L. (2005). Extensión universitaria en el escenario comunitario. Conceptualización y difusión de una experiencia. Tesis doctoral, Universidad Agraria de La Habana.

Pérez, L. (2007). Pertinencia y extensión universitaria en el contexto de la nueva universidad cubana. Revista Pedagogía Universitaria, 12 (1).

Santamarta, J. (s/f). ¿Qué es la globalización? Consultado el 10 de enero de 2014 en (http://www.plades.org.pe/etextos/santamarta.htm).

Segredo, A. (2011). La gestión universitaria y el clima organizacional. Educación Médica Superior, 25 (2), 164-177.

Smith, N. (2001). Nuevo globalismo, nuevo urbanismo. Documentos de análisis geográficos, 38, 15-32.

UNESCO. (2009). Conferencia Mundial de la Educación Superior. París.

Vallaeys, F. (2008). ¿Qué es la responsabilidad Social Universitaria? Consultado en noviembre de 2012 en: http://palestra. pucp.edu.pe/index.php?id=111 